\title{
UNSUPERVISED TEXTURE CLASSIFICATION OF ENTROPY BASED LOCAL DESCRIPTOR USING K-MEANS CLUSTERING ALGORITHM
}

\author{
S. S. Sreeja Mole ${ }^{1)}$, L. Ganesan 2) \\ 1) Department of Computer Science and Engineering, \\ Government College of Engineering, Tirunelveli, \\ Tamil Nadu, India \\ sreejamole@yahoo.com \\ ${ }^{2)}$ Department of Computer Science and Engineering, \\ A. C. College of Engineering and Technology, Karaikudi, \\ Tamil Nadu, India \\ drlgtvly@yahoo.com
}

\begin{abstract}
This paper presents an efficient approach for unsupervised Texture Segmentation and Classification, based on features extracted from entropy based local descriptor using K-means clustering with spatial information. The Kmeans clustering algorithm is commonly used in computer vision as a form of image segmentation. Texture analysis refers to a class of mathematical procedures and models that characterizes the spatial variations within imagery as a means of extracting information. Texture analysis may require the solution of two different problems first is Segmentation and Classification of a given image according to the different texture and second was for of a given texture with respect to a set of known textures. Based on the proposed concept, this paper describes the entropy based local descriptor using K-Means with spatial information approach. Experimental results show that the proposed framework performs very well compared to other clustering algorithms in all measured criteria. Spatial information has been effectively used for unsupervised texture classification for Brodatz of texture images. The model is not specifically confined to a particular texture feature. We tested this algorithm using other texture features. The proposed entropy based local descriptor approach gives good accuracy when compared with other methods.
\end{abstract}

Keywords: Texture- Local Binary Pattern operator, Unsupervised texture classification, Entropy based Local Descriptor, K-Means clustering algorithm.

\section{INTRODUTION}

The goal of texture analysis is to produce a classification map of the input image where each uniform textured region is identified with the texture class. Texture plays an important role in low level image analysis and understanding. The gray-level co-occurrence matrix approach is one of the most popular statistical methods used in practice to measure the textural information of images. Textures may vary according to randomness, regularity (or periodicity), directionality and orientation. Texture is an important spatial feature, useful for identifying objects or regions of interest in an image. The word texture is used to refer to a number of commonly encountered visual patterns in natural scenes such as forlage, grass, pebbles, clouds etc, while there is no proper definition of texture, it is widely accepted that the term generally refers to a reputation of certain basic elements sometimes called texels. Image analysis essentially affects the overall performance of any automated image analysis system thus its quality is of the utmost importance. Texture Segmentation and Classification are the fundamental process which partitions a data space into meaningful salient regions. Image regions, homogeneous with respect to some usually textural or color measure, which result from a classification algorithm are analyzed in subsequent interpretation steps.

Texture based image classification is area of intense research activity in the past thirty years and many algorithms were published in consequence of all this effort, starting from simple threshold methods up to the most sophisticated random field type methods. Image segmentation and classification plays an important role in a variety of applications such as robot vision, object recognition, and medical imaging. Texture images are analyzed by identifying the local and global properties of the images under consideration. 
Unsupervised methods which do not assume any prior scene knowledge which can be learned to help classification process are obviously more challenging than the supervised ones. Texture classification is a key field in many computer vision applications ranging from quality control to remote sensing. The problem of feature selection is defined as follows given a set of candidate features; select a subset that performs the best under classification system. The procedure can reduce the cost of recognition and provide better classification accuracy. There are two general approaches to segmenting images with intensity inhomogeneities. The first approach is to separately apply a correction algorithm, followed by a segmentation algorithm. This approach allows exibility in that once the image has been corrected, the intensity in homogeneities can essentially be ignored in any subsequent processing. The second approach used to segment images with intensity in homogeneities is to simultaneously compensate for the shading effect while segmenting the image. This approach has the advantage of being able to use intermediate information from the segmentation while performing the correction. Image classification systems have received a recent boost from methods using local features generated over interest points, delivering higher robustness against partial occlusion and cluttered backgrounds. There are two widely used approaches to describe the texture of a region, these are statistical and structural. The statistical approach consolers that the intensities are generated by a two dimensional random field and examples of statistical approaches of texture analysis are auto correlation function, gray level co occurrence methods, Fourier texture analysis, edge frequency and law's texture energy measures. The structural techniques deal with the arrangement of image primitives such as the description of texture based on regularly spaced parallel lines. Statistical methods analyze the spatial distribution of gray values by computing local features at each point in the image and deriving a set of statistics from the distributions of the local features. The use of statistical features is one of the early methods proposed in the research literature [The reason behind that is the fact that the spatial distribution of gray values is one of the defining qualities of texture. Statistical methods can be classified into first-order (one pixel), second-order (two pixels) and higher-order (three or more pixels) statistics based on the number of pixels defining the local feature [1].The first order statistics estimate properties like the average and variance of individual pixel values, ignoring the spatial interaction between image pixels, secondand higher-order statistics on the other hand estimate properties of two or more pixel values occurring at specific locations relative to each other. Co-occurrence features and gray level differences are the most widely used statistical methods, which inspired a variety of modifications later on [1],[11] including signed differences and the Local Binary Pattern (LBP) operator. LBP operator combines statistical and structural approaches to texture analysis by incorporating occurrence statistics of simple local microstructures.

In this paper Entropy based LBP method is used to extract the features from the images to perform Unsupervised Classification. This paper is organized as follows. In section 2, the theory of Entropy is reviewed. The LBP is explained in section 3. In section 4, Unsupervised Entropy based Local Descriptor operator classification algorithm is reviewed. Experiments results are shown in section 5 and finally conclusion is on section 6 .

\section{ENTROPY}

Entropy, as defined by Clausius, is the true cornerstone of equilibrium thermodynamics.

Entropy was chosen as a measure of the detail provided by each picture. The word "entropy" was created by Rudolf Clausius and it appeared in his work "Abhandlungen "uber die mechanische W"armetheorie" published in 1864. The word has a Greek origin, its first part reminds us of "energy" and the second part is from "tropos" which means turning point. Clausius' work is the foundation stone of classical thermodynamics. According to Clausius, the change of entropy of a system is obtained by adding the small portions of heat quantity received by the system divided by the absolute temperature during the heat absorption. This definition is satisfactory from a mathematical point of view and gives nothing other than an integral in precise mathematical terms. Clausius postulated that the entropy of a closed system cannot decrease, which is generally referred to as the second law of thermodynamics. The Entropy[7] is defined as the average number of binary symbols necessary to code a given input given the probability of that input appearing an a stream. High entropy is associated with a high variance in the pixel values, while low entropy indicates that the pixel values are fairly uniform, and hence little detail can be derived from them. Therefore, when applied to groups of pixels within the source images, entropy provides a way to compare regions from the different source images. Every pixel in the final image is computed as the weighted average of the corresponding pixels in the source images where each value is weighted by the entropy of the 
surrounding region. For each pixel $p=(u, v)$ in the final image there are corresponding pixels $\left(\mathrm{p}_{1}, \mathrm{p}_{2}\right.$, $\ldots, \mathrm{p}_{\mathrm{N}}$, ) one for each source image. For each pixel pi in each image, the local entropy (measured within a fixed window) vi is computed, and the weighted average $\mathrm{p}$ is computed.The entropy for the pixel window is computed as,

$$
\text { Entropy }=-\sum p(i, j) \log p(i, j)
$$

Where $\mathrm{P}(\mathrm{i}, \mathrm{j})$ - Probability value if the $i^{\text {th }}$ and $j^{\text {th }}$ pixel in the window.

The steps involved in texture classification is shown in Fig. 1.

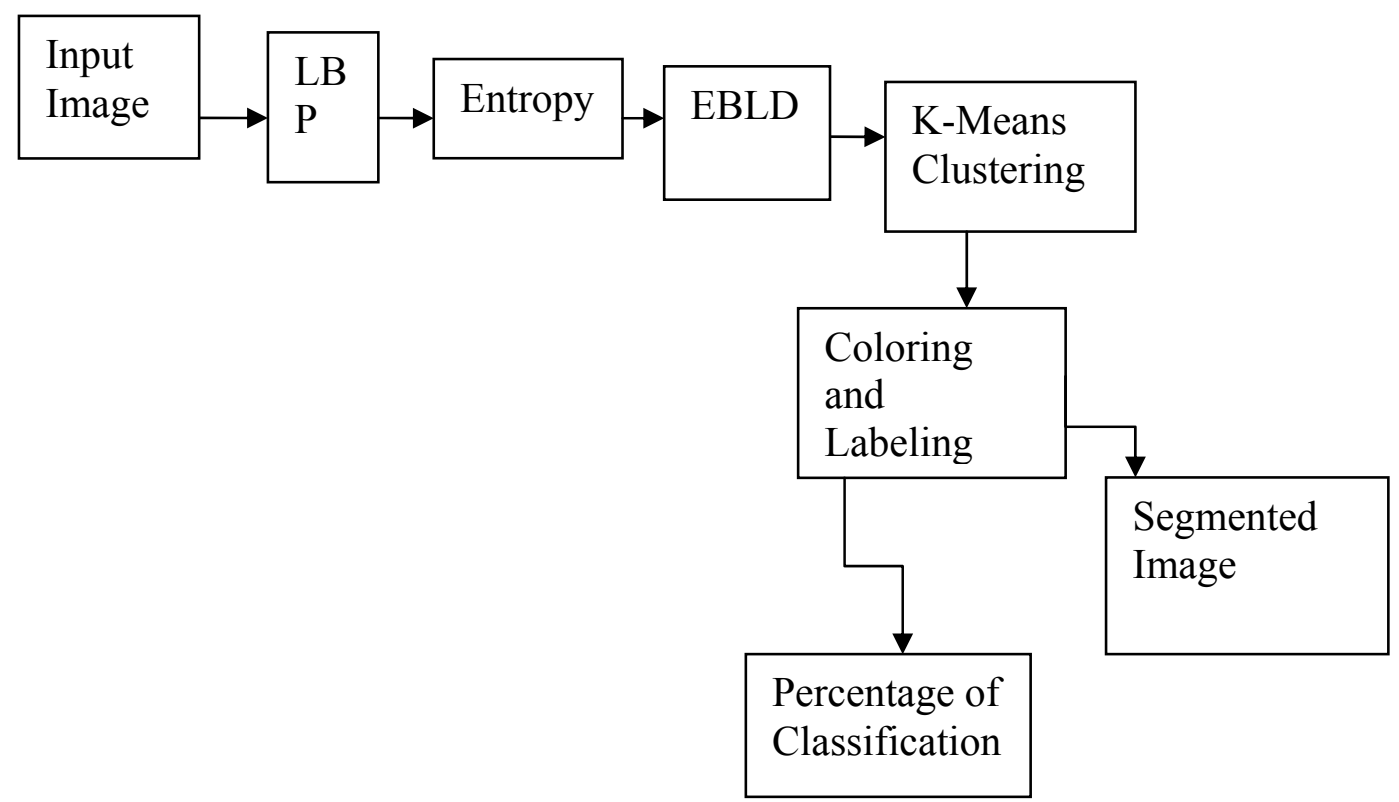

Fig. 1 - Step in Texture Classification

\section{LOCAL BINARY PATTERN OPERATOR}

In Local Binary Pattern Operator method $[5,6]$ uses the operators with eight neighboring pixels using the center as a threshold by multiplying the threshold values by weights given by powers of two. By definition Local Binary Pattern Operator is invariant to any monotonic transformation of the gray scale and its quick to compute with larger neighborhoods, the number of possible Local Binary Pattern Operator codes increased exponentially. This can be avoided to some extent by considering only a subset of that codes one approach is to use so called uniform patterns representing the statistically most Local Binary Pattern Operator. Local binary pattern operator method is described with 28 possible texture units. The texture unit is obtained by applying the threshold operation using the following rule in equation 1 .

$$
E_{i}=\left\{\begin{array}{l}
0, V_{i}<V_{0} \\
1, V_{i}>V_{0}
\end{array}\right.
$$

Where $V_{0}$ is the center pixel and $V_{i}$ is the intensity value of the neighboring pixel $i$.

The Local Binary Pattern Operator is determined as

$$
\text { Local Binary Pattern Operator }=\sum_{I=1}^{8} E_{i} 2^{i-1}
$$

Local Binary Pattern Operator does not take into account the contrast of texture which is the measure of local variations present in an image and is important in the description of some textures. Texture spectrum operator is similar to LBP Operator but it uses three levels that is, two thresholds instead of two levels used in Local Binary Pattern Operator. This leads to a more efficient representation and implementation than with Local Binary Pattern Operator.

\section{ENTROPY BASED LOCAL DESCRIPTOR}

The input image is reconstructed using LBP. After reconstruction, Entropy values of reconstructed image are taken as the texture features. 


\subsection{ALGORITHM FOR ENTROPY WITH K-MEANS CLUSTERING TECHNIQUES}

$\mathrm{K}$-means is one of the simplest unsupervised learning algorithms that solve the well known clustering problem[1]. The procedure follows a simple and easy way to classify a given data set through a certain number of clusters (assume $\mathrm{k}$ clusters) fixed a priori. The method developed for this task, though simple, is both flexible and powerful. The K-means algorithm does not necessarily find the most optimal configuration, corresponding to the global objective function minimum. The algorithm is also significantly sensitive to the initial randomly selected cluster centers. The K-means algorithm can be run multiple times to reduce this effect. K-means is a simple algorithm that has been adapted to many problem domains.

1. Input the texture mosaic image with size $\mathrm{m} x \mathrm{n}$.

2. Take a window of size $30 \times 30$ in the image and find the value of Entropy using the below

Equation as Entropy $=-\Sigma$ pi $\log$ (pi)

$\mathrm{Pi}$ - Probability value if the ith pixel in the window

3. Move the window towards the whole image by using overlapping method and find the Entropy for all the windows.

4. Cluster the all Entropy values using K Means clustering techniques.

5. Label the different clusters and Colour the different labels.

6. Output the classified image.

\subsection{ALGORITHM FOR ENTROPY BASED LOCAL DESCRIPTOR WITH K-MEANS TECHNIQUES}

1. Input the texture mosaic image with size $\mathrm{m} x \mathrm{n}$.

2. Take a window of size $30 \times 30$ in the image and reconstruct the window by LBP using the following steps.

i. Take a sub-window of size $3 \times 3$ in the window image.

ii. Compare the all pixel values of the window with the centre pixel.

iii. Note the difference: If pixel value less than centre pixel value, note the difference is 0 . If pixel value greater than or equal to centre pixel value, note the difference is 1 .

iv. Form a eight digit binary value from the noted difference values (totally 8 pixels except centre pixel).

v. Find the grey value of the eight digit binary value.

vi. Replace the pixel of the window image using the grey value. vii. Move the sub-window towards the whole window image using overlapping method and find the grey value of the all sub-windows using above mentioned Entropy Based Local Descriptor method and replace the all pixels of the window image using these grey values.

viii. Return the Reconstructed Entropy Based Local Descriptor window image.

3. Find the value of entropy based local descriptor for reconstructed Entropy Based Local Descriptor window using the below equation as Entropy $=-\Sigma$ pi log (pi)

$\mathrm{Pi}$ - Probability value if the ith pixel in the window. Move the window towards the whole image by using overlapping method and find the entropy based local descriptor value for all the windows.

4. Cluster the all entropy based local descriptor values using $\mathrm{K}$ Means clustering techniques.

5. Label the different clusters and Color the different labels.

6. Output the segmented and classified image.

The classification of texture image in the input mosaic image is calculated by the following equation Classification Percentage $=$ (number of pixels classified in a texture image / total number of pixels in a texture image)*100.

\section{EXPERIMENTAL RESULTS}

Experiments are conducted with two,four and six monochrome texture images each of size $30 \times 30$, obtained from Brodatz album and are shown in Figure. 2. The overall classification results are shown in Table.1, Table 2 and Table 3. In order to evaluate the K-Means Clustering algorithm classification method with the LBP, Entropy Operator and EBLD, the set of images have been taken. The input images respectively D1, D2 D5, D6, D20, D21, D17, D21, D49, D53, D55 and D60 are shown in Figure 3.13(a). The output image after applying unsupervised K-Means Clustering classification methods in LBP, Entropy and EBLD are shown in Figure.

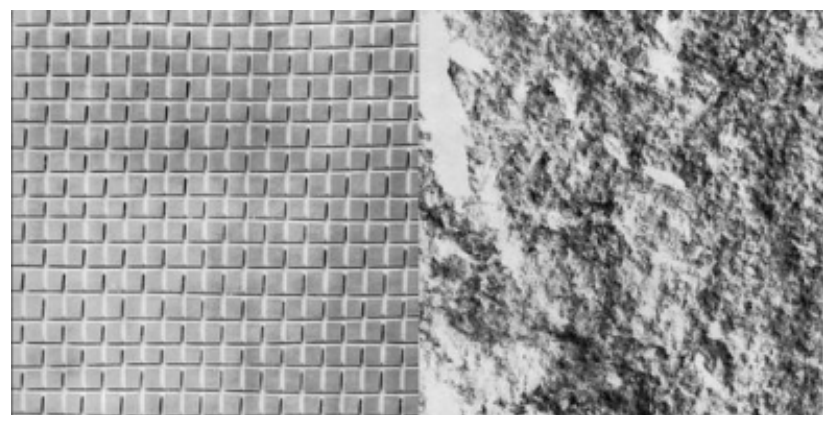

(a) D1, D2 Input Image 


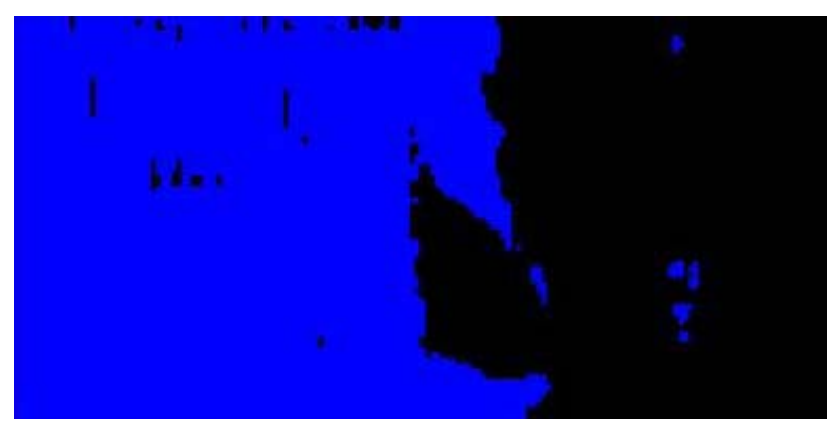

(b) LBP Operator

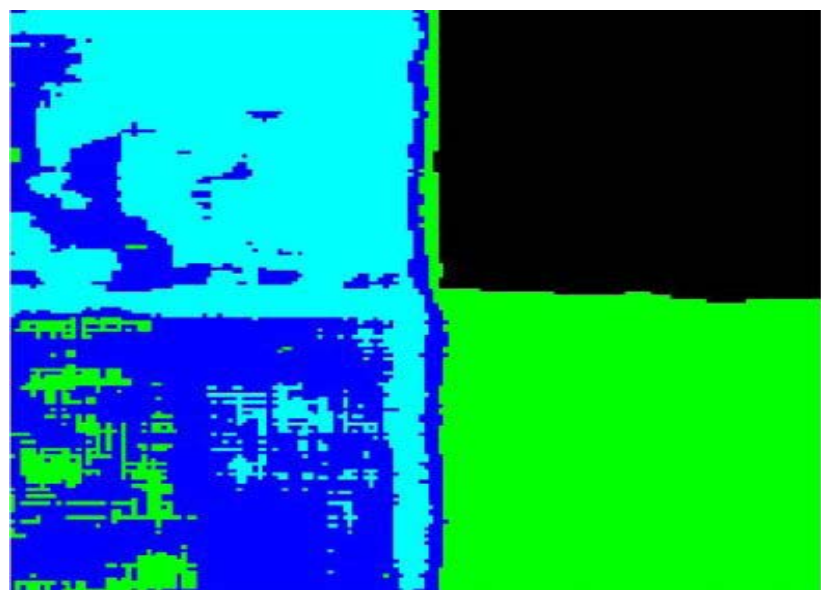

(b) LBP Operator

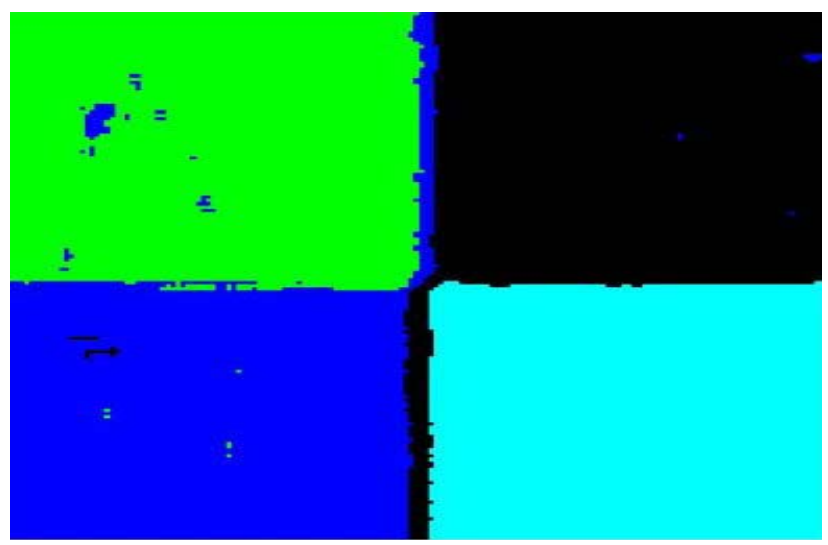

(c) Entropy Operator

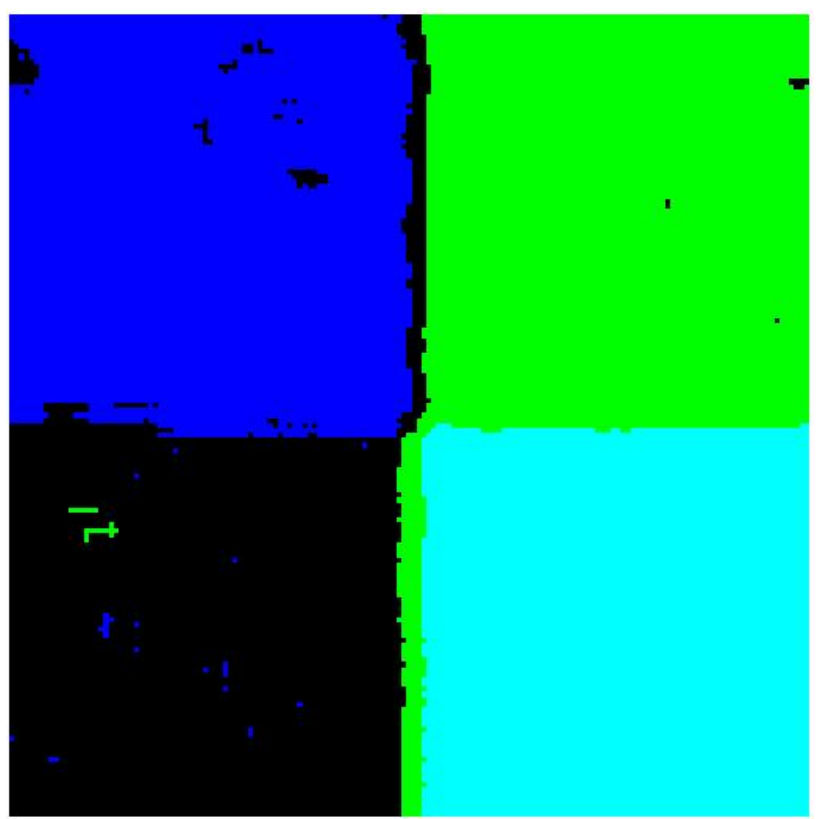

(d) Entropy Based Local Descriptor

(a) D5, D6, D20, D21 Input Image 


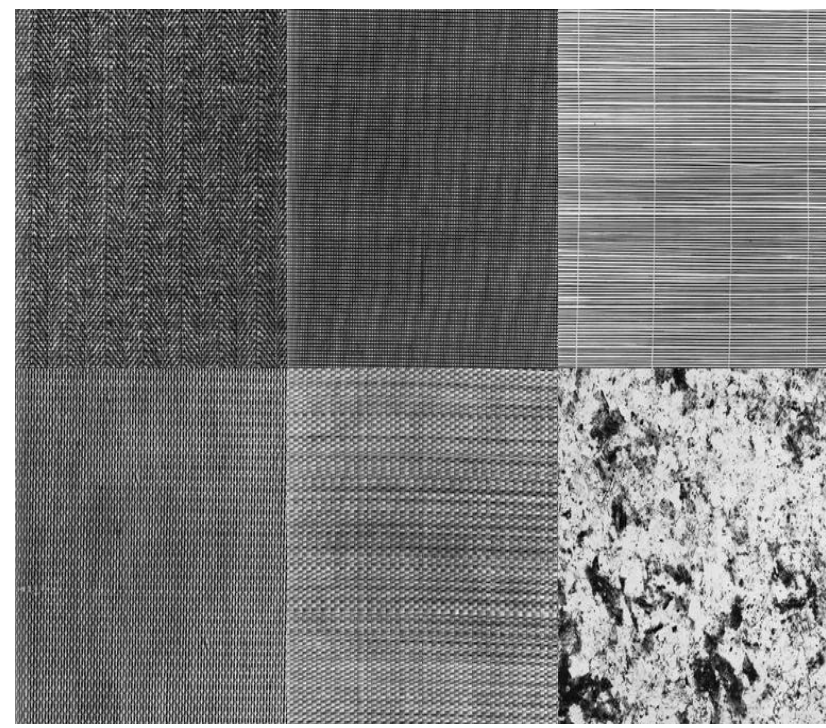

(a) D17, D21, D49, D53, D55 and D60 Input Image

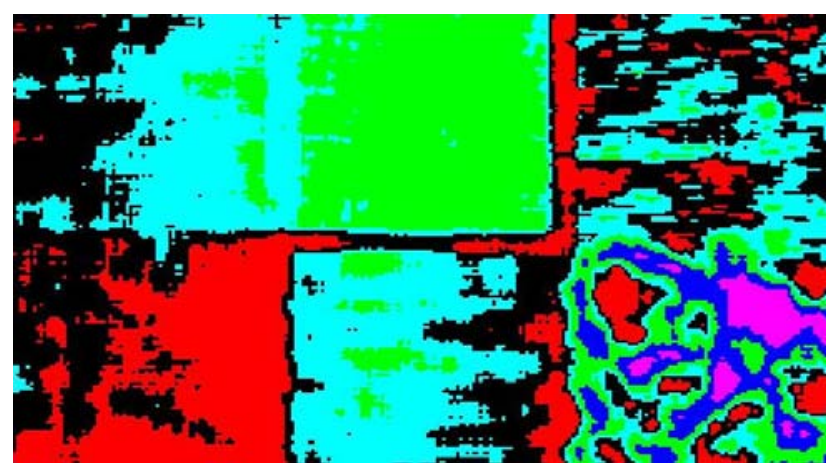

(b) LBP Operator

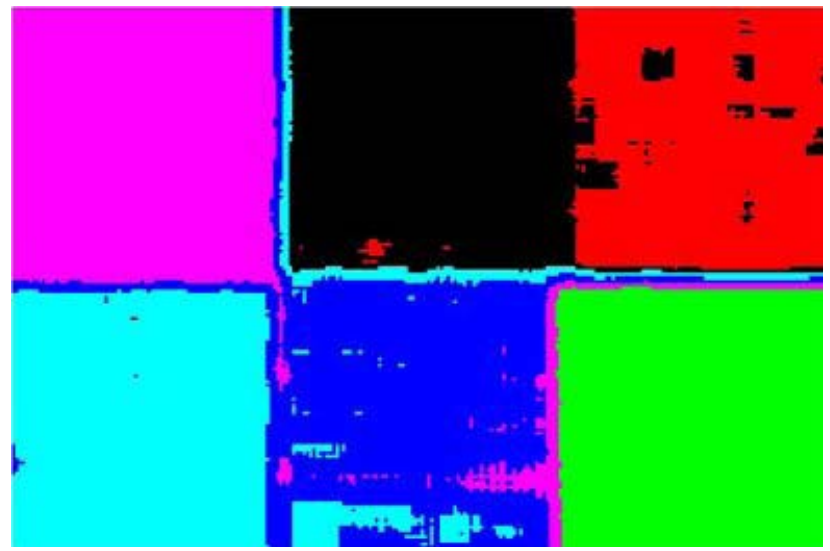

(c) Entropy Operator

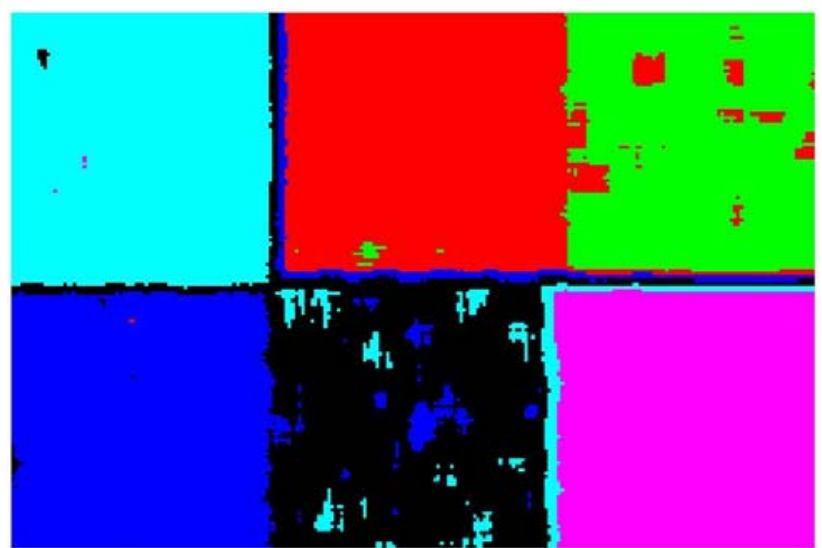

(d) Entropy Based Local Descriptor

Fig. 2 - (a),(b), (c) and (d) Unsupervised Texture Segmentation of Brodatz Images using K-Means Method

Table 1. Results for Unsupervised Texture classification of Two Brodatz Images using K-Means Clustering method

\begin{tabular}{|l|l|l|l|l|}
\hline \multirow{3}{*}{ Image } & \multicolumn{3}{l}{ Classification Percentage (\%) } \\
\cline { 2 - 5 } & \multicolumn{2}{|l|}{ Entropy Operator } & \multicolumn{2}{l|}{$\begin{array}{l}\text { Entropy Based Local Descriptor with } \\
\text { K-means }\end{array}$} \\
\cline { 2 - 5 } & D1 & D2 & D1 & D2 \\
\hline D1 & 98.03 & 1.97 & 98.07 & 1.93 \\
\hline D2 & 13.31 & 86.69 & 0.21 & 99.79 \\
\hline
\end{tabular}

Table 2. Results for Unsupervised Texture classification of Four Brodatz Images using K-Means Clustering method

\begin{tabular}{|c|c|c|c|c|c|c|c|c|}
\hline \multirow{3}{*}{ Image } & \multicolumn{8}{|c|}{ Classification Percentage (\%) } \\
\hline & \multicolumn{4}{|c|}{ Entropy Operator } & \multicolumn{4}{|c|}{$\begin{array}{l}\text { Entropy Based Local Descriptor with } \\
\text { K-means }\end{array}$} \\
\hline & D5 & D6 & D20 & D21 & D5 & D6 & D20 & D21 \\
\hline D5 & 81.98 & 0 & 17.82 & 0.19 & 98.55 & 0 & 1.45 & 0 \\
\hline D6 & 2.06 & 92.79 & 0.21 & 4.95 & 0 & 95.46 & 3.88 & 0.66 \\
\hline D20 & 17.02 & 0 & 70.88 & 12.10 & 2.25 & 3.63 & 94.12 & 0 \\
\hline D21 & 2.43 & 0.09 & 4.04 & 93.44 & 0 & 6.48 & 0.08 & 93.44 \\
\hline
\end{tabular}


Table 3. Results for Unsupervised Texture classification of Six Brodatz Images using K-Means Clustering method

\begin{tabular}{|c|c|c|c|c|c|c|c|c|c|c|c|c|}
\hline \multirow{3}{*}{ Image } & \multicolumn{12}{|c|}{ Classification Percentage (\%) } \\
\hline & \multicolumn{6}{|c|}{ Entropy Operator } & \multicolumn{6}{|c|}{ Entropy Based Local Descriptor with K-means } \\
\hline & D17 & D21 & D49 & D53 & D55 & D60 & D17 & $\mathrm{D} 21$ & D49 & D53 & D55 & D60 \\
\hline D17 & 53.72 & 0 & 0.87 & 6.42 & 38.99 & 0 & 93.85 & 0 & 0.72 & 0 & 0 & 5.42 \\
\hline D21 & 0 & 81.18 & 4.51 & 0 & 13.04 & 1.27 & 0.09 & 92.26 & 0 & 5.26 & 2.39 & 0 \\
\hline D49 & 0 & 32.80 & 49.48 & 14.08 & 3.63 & 0.01 & 0.44 & 0 & 52.24 & 1.85 & 9.08 & 36.39 \\
\hline D53 & 0 & 1.09 & 0 & 62.94 & 0 & 35.97 & 10.46 & 0 & 0.47 & 89.00 & 0 & 0.07 \\
\hline D55 & 0 & 7.06 & 7.39 & 28.89 & 56.66 & 0 & 0 & 11.16 & 2.50 & 0 & 86.34 & 0 \\
\hline D60 & 24.39 & 13.72 & 12.10 & 10.37 & 14.37 & 25.03 & 0 & 0.40 & 1.97 & 0 & 0 & 97.63 \\
\hline
\end{tabular}

The unsupervised texture analysis yields the result of $98.03 \%$ of classification when entropy based algorithm is used and $98.07 \%$ of classification when the entropy based local descriptor with KMeans is used for D1 image. In the case of D2 image there is only $86.69 \%$ of classification when entropy based algorithm is used while entropy based local descriptor with K-Means yields a higher classification of $99.79 \%$.The unsupervised texture analysis yields the result of $81.98 \%$ of classification when entropy based algorithm is used and $98.55 \%$ of classification when the entropy based local descriptor with K-Means is used for D5 image. In the case of D6 image there is only $92.79 \%$ of classification when entropy based algorithm is used while entropy based local descriptor with K-Means yields a higher classification of $95.6 \%$.In the case of D20 image there is only $70.88 \%$ of classification when entropy based algorithm is used while entropy based local descriptor with K-Means yields a higher classification of $94.12 \%$.In the case of D21 image there is only $93.44 \%$ of classification when entropy based algorithm is used while entropy based local descriptor with K-Means yields a higher classification of $93.44 \%$. The unsupervised texture analysis yields the result of $53.72 \%$ of classification when entropy based algorithm is used and $93.85 \%$ of classification when the entropy based local descriptor with K-Means is used for D17 image. In the case of D21 image there is only $81.18 \%$ of classification when entropy based algorithm is used while entropy based local descriptor with K-Means yields a higher classification of $92.26 \%$.In the case of D49 image there is only $49.48 \%$ of classification when entropy based algorithm is used while entropy based local descriptor with K-Means yields a higher classification of $52.24 \%$.In the case of D53 image there is only $62.94 \%$ of classification when entropy based algorithm is used while entropy based local descriptor with K-Means yields a higher classification of $89.00 \%$.In the case of D55 image there is only $56.66 \%$ of classification when entropy based algorithm is used while entropy based local descriptor with K-Means yields a higher classification of $86.34 \%$.In the case of D60 image there is only $25.03 \%$ of classification when entropy based algorithm is used while entropy based local descriptor with K-Means yields a higher classification of $97.63 \%$.

\section{CONCLUSION}

In this paper, entropy based local descriptor features are extracted and successfully implemented for unsupervised classification. This method is tested with publicly available Brodatz images. The algorithm performs well under different Gaussian noise levels. The results of the proposed method are compared with the existing method. The entropy based local descriptor method gives the better efficient results for classification. The experiments show that the proposed method can achieve better results than existing unsupervised texture classification approaches. The proposed entropy based local descriptor approach gives good accuracy when compared with other methods. The effect of changing textures on the texture analysis system performance is under current investigation and the research work is under progress.

\section{ACKNOWLEDGMENT}

The authors are more thankful to the Principal and Head of the Department of Computer Science and Engineering of our College for their constant support and encouragement. The authors also extend their gratitude to the anonymous reviewers who have given very good suggestions for this better presentation of our manuscript.

\section{REFERENCES}

[1] Timo Ojala T., Pietikainen M. and Maenpaa T. Multiresolution Gray Scale and Rotation Invariant Texture Classification with Local Binary Patterns. IEEE Transactions on Pattern 
Analysis and Machine Intelligence, (24) 7 (2002). pp. 65-70.

[2] Yong Hu, Chun-xia Zhao. Unsupervised Texture Classification by Combining Multiscale Features and K-means Classifier. (2009). IEEE.

[3] R. M. Haralick, K. Shanmugan and I. Dinstein. Textural features for image classification. IEEE Trans. Syst. Man, Cybern., (vol. SMC-3) no. 6 (1973). pp. 610-621.

[4] R. M. Haralickю Statistical and structural approaches to textureю in Proc. IEEE, (67) 5 (1979). pp. 786-804.

[5] $\mathrm{He}$ and $\mathrm{Li}$ Wang. Texture unit, Texture spectrum and texture analysis. IEEE Transaction on Geo Science and Remote sensing, (28) 4 (1990). pp. 509-512.

[6] Hui Zhou, Runsheng Wang, Cheng Wang. A novel extended local-binary-pattern operator for texture analysis. Information Sciences, (178) (2008). pp. 4314-4325.

[7] Shu Liao and Albert C. S. Chung. Texture classification by using advances local binary patterns and spatial distribution of dominant patterns. ICASSP 2007, pp. 1221-1224.

[8] M.C. Padma et al. Entropy Based Texture Features Useful for Automatic Script Identification. (IJCSE) International Journal on Computer Science and Engineering, (2) 2 (2010). pp. 115-120.

[9] D. C. He, L. Wang, and J. Guibert. Texture features extraction. Pattern Recogn. Lett., (6) (1987). pp. 269-273.

[10] L. Van Gool, P. Dewaele and A. Oosterlinck. Survey-texture analysis Anno 1983. Comput.
Vision, Graphics \& Image Process., (29) (1985). pp. 336-357.

[11] Ojala T and Pietikäinen M. Unsupervised Texture Segmentation Using Feature Distributions. Pattern Recognition, (32) (1999). pp. 477-486.

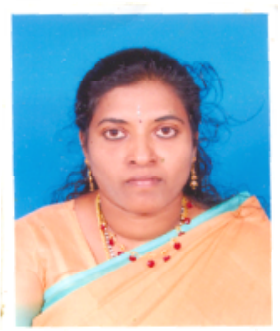

S. S. Sreeja Mole completed her B.E in Electronics and Communication Engg and M.E in Communication Systems. from Thiagaraja College of Engineering, Madurai. She has authored more than five publications in reputed International Journals. She is doing Ph.d in image processing. Her area of interest includes image processing, multimedia and compressions. She is currently working as lecturer in department of Computer science and engineering, Government College of Engg, Tirunelveli.

Dr. L.Ganesan completed his B.E in Electronics and Communication Engg. from Thiagaraja College of Engineering, Madurai and M.E in Computer Science and Engg. from Government College of Technology, Coimbatore. He completed his Ph.D from Indian Institute of Technology, Kharapur in the area image processing. He has authored more than fifty publications in reputed International Journals. His area of interest includes image processing, multimedia and compressions. He is currently working as head of the department in Computer science and engineering, A.C. College of Engg. and Technology, Karaikudi. 